\title{
Kinesiólogos frente a la pandemia de COVID-19: ¿Cuál es su rol?
}

\author{
L. FELIPE DAMIANI R.*, YORSCHUA JALIL C.**,***, ROQUE BASOALTO E.**,****, \\ GREGORY VILLARROEL S.* y PATRICIO GARCÍA V.*
}

\section{Physiotherapists in COVID-19: what is their role?}

The impact of the COVID-19 pandemic on the social, economic, and health levels is unprecedented. In Chile alone until July 2020, more than 340,000 people have tested positive and around 9,000 have died because of it. This health crisis has led to different adaptations in the health system and extraordinary measures have been taken to meet these needs. The physiotherapist as a health professional constitutes a fundamental piece in the care of these patients. Its role has been reflected in all disease's stages, from the emergency room to the intensive care unit and after hospital discharge. In addition to his clinical-attendance role, the physiotherapist may contribute in different areas such as education, health promotion, health management, and scientific research, aspects that could be essential in managing the pandemic. As physiotherapist responsibility, we should consider this opportunity and assume the multiple challenges derived from the pandemic to commit and provide answers to current health needs.

Key words: COVID-19; Physiotherapist; Rehabilitation.

\section{Resumen}

El impacto de la pandemia por COVID-19 a nivel social, económico y sanitario no tiene precedentes. Sólo en Chile hasta el mes de julio de 2020, más de 340.000 personas han contraído la enfermedad y alrededor de 9.000 han fallecido por esta causa. Esta crisis sanitaria ha llevado a una adaptación en todo el sistema de salud y toma de medidas extraordinarias para poder cubrir dichas necesidades. El kinesiólogo como profesional de la salud constituye una pieza fundamental en la atención de estos pacientes. Su rol se ha visto reflejado en las distintas etapas de la enfermedad desde la atención primaria y urgencia hasta la atención de pacientes en la unidad de cuidados intensivos y posterior al alta hospitalaria. Además de su rol clínico asistencial, el kinesiólogo es capaz de contribuir en áreas de educación, promoción, gestión en salud e investigación científica, aspectos que podrían ser esenciales en el manejo de la pandemia. Es deber del kinesiólogo considerar esta oportunidad y asumir los múltiples desafios derivados de la pandemia para comprometerse y otorgar respuestas a las necesidades sanitarias actuales.

Palabras clave: COVID-19; Kinesiólogo; Rehabilitación.

\footnotetext{
* Departamento Ciencias de la Salud, Carrera de Kinesiología, Facultad de Medicina, Pontificia Universidad Católica de Chile. Santiago, Chile.

** Programa de Doctorado en Ciencias Médicas, Facultad de Medicina, Pontificia Universidad Católica de Chile. Santiago, Chile.

*** Escuela de Kinesiología, Facultad de Ciencias de la Rehabilitación, Universidad Andrés Bello. Santiago, Chile.

**** Departamento Medicina Intensiva. Facultad de Medicina, Pontificia Universidad Católica de Chile. Santiago, Chile.
} 


\section{Introducción}

El nuevo coronavirus (SARS-CoV-2) reportado en diciembre del año 2019 en China, ha sido responsable de una pandemia con consecuencias sanitarias, sociales y económicas incalculables. La rápida propagación del virus ha ocasionado que, hasta julio del 2020, existan más de 16 millones de personas contagiadas en 188 países, causando la muerte en más de 650.000 de ellas ${ }^{1}$. En Chile, al 26 de julio, se han confirmado un total de 345.790 casos, llegando a 9.112 fallecidos desde que se confirmó el primer caso, el 3 de marzo de $2020^{2}$.

La crisis sanitaria por COVID-19 ha llevado a una adaptación de todo el sistema de salud en nuestro país incluyendo la expansión de camas críticas, modificación de jornadas laborales del personal de salud, reconversión de camas en distintos servicios y unidades hospitalarias, así como también incremento del personal de salud especializado y no especializado, entre los que destaca el kinesiólogo como profesional importante del equipo de salud en la atención de estos pacientes. El objetivo de esta perspectiva es describir los diversos roles y potenciales ámbitos de acción donde el kinesiólogo contribuye en el contexto de esta pandemia.

\section{Principales alteraciones respiratorias y físicas de pacientes con COVID-19}

$\mathrm{La}$ actual enfermedad por coronavirus (COVID-19) tiene un espectro variable de presentación, desde una evolución asintomática hasta un cuadro clínico grave por insuficiencia respiratoria aguda. Las alteraciones y complicaciones de pacientes con insuficiencia respiratoria por COVID-19 son diversas y dependen de la fase de la enfermedad y el soporte o asistencia que requieran ${ }^{3}$.

En etapas tempranas de la enfermedad, la mayoría de los síntomas y signos observados están relacionados al desarrollo de una insuficiencia respiratoria aguda. Así, los pacientes pueden presentar aumento del trabajo respiratorio, representado por aumento de la frecuencia respiratoria, uso de musculatura respiratoria accesoria, alteración del intercambio gaseoso, disminución de la ventilación y fatiga muscular respiratoria ${ }^{4}$.

Si la enfermedad progresa a etapas más severas, una proporción de pacientes (5-10\%) requerirá admisión a la unidad de cuidados intensivos (UCI) para monitoreo y eventual uso de ventilación mecánica invasiva (VM). En este punto, múltiples complicaciones podrían emerger relacionadas al uso de VM, drogas sedantes e inmovilismo tales como daño pulmonar y diafragmático inducido por ventilación mecánica, desarrollo de atrofia y debilidad muscular, junto con una disminución de la función cardiovascular y pulmonar ${ }^{5,6}$. La presencia de estas complicaciones ha sido asociada a una prolongación de la estadía hospitalaria y contribuye al aumento de morbimortalidad y costos de salud ${ }^{7,8}$.

Finalmente, existe una etapa desconocida de la enfermedad que se manifiesta posterior a la UCI y que sin duda constituye un problema de salud importante. La evidencia indica que aquellos pacientes que sobreviven a la fase crítica de una enfermedad en la UCI persisten con atrofia y debilidad muscular generalizada ${ }^{5,9}$, presentando una capacidad limitada de desarrollar actividades de la vida diaria hasta 5 años posterior al alta hospitalaria, con un gran costo personal, social $\mathrm{y}$ económico ${ }^{7}$. En pacientes con COVID-19, se ha demostrado una disminución de la función pulmonar y la existencia de síntomas tales como fatiga, disnea y dolor articular posterior al evento agudo en más del $60 \%, 40 \%$ y $20 \%$ de los pacientes respectivamente ${ }^{10,11}$. A pesar de la limitada evidencia actual, es razonable pensar que aquellos pacientes recuperados y que hayan sobrevivido a un evento crítico por COVID-19 presentarán diversas limitaciones en la función respiratoria, capacidad física y calidad de vida $^{7,11,12}$. En este sentido, como profesionales de la salud enfrentamos un desafío complejo que requiere la organización e integración de diferentes conocimientos y habilidades que aporten en el manejo, optimización y control de esta pandemia.

\section{Rol del kinesiólogo en la pandemia COVID-19}

El kinesiólogo como profesional de la salud cumple un rol importante en la batalla contra el COVID-19. Tradicionalmente, se ha destacado por su rol clínico asistencial durante todo el ciclo vital, no obstante, su ámbito de acción no está limitado únicamente a ello. Su formación, la cual ha evolucionado en los últimos años, ha ampliado sus competencias profesionales y contextos de desempeño. Su contribución en esta pandemia, en conjunto con un equipo interdisciplinario, se ve reflejada en todos los niveles de atención en salud, desde la atención primaria, residencias sanitarias, urgencias, salas hospitalarias básicas, UCI y seguimiento posterior al alta hospitalaria ${ }^{13,14}$. 
El abordaje kinesiológico clínico asistencial en las diferentes fases de la enfermedad contempla 3 principales dimensiones: i) terapia ventilatoria, destinada a la evaluación, implementación y manejo de soportes ventilatorios como la ventilación mecánica (invasiva y no invasiva), oxigenoterapia convencional y terapias de alto flujo (cánula nasal de alto flujo), sistemas de humidificación de la vía aérea (activa y pasiva) y descontinuación la ventilación mecánica ${ }^{15,16}$; ii) intervención kinesiológica respiratoria, a través de la aplicación de técnicas instrumentales y no instrumentales que promueven la higiene bronquial y favorecen la ventilación y optimización del intercambio gaseoso a través de la posición prono ya sea en pacientes vigiles o intubados y ventilados mecánicamente acelerando su recuperación ${ }^{17,18} ; \mathrm{y}$ iii) Intervención kinesiológica funcional, a través del fortalecimiento muscular y readaptación al esfuerzo físico, que le permite a los pacientes evitar atrofia muscular, retornar a un estatus funcional independiente, favoreciendo la realización de actividades básicas de la vida diaria (alimentarse, vestirse, caminar, etc.) y por tanto, mejorando su calidad de vida ${ }^{19,20}$.

Del mismo modo, otros roles tales como educación, prevención, promoción, gestión e investigación en salud durante todo el curso de la enfermedad son cruciales para un manejo oportuno y control adecuado de esta pandemia.

En Chile, durante la pandemia por COVID-19, el número de camas críticas aumentó en aproximadamente un $300 \%$, causando la incorporación de profesionales no especialistas en la atención de pacientes. En este sentido, la capacitación y formación de kinesiólogos y otros profesionales de la salud por medio de sus pares experimentados $\mathrm{y}$ calificados constituyen un elemento fundamen$\mathrm{ta}^{21}$. En línea con su rol educativo, el kinesiólogo (principalmente en atención primaria) juega un papel clave en el manejo de la enfermedad, reconocimiento de signos y síntomas de exacerbación respiratoria, adecuado uso de fármacos y adherencia al tratamiento. Además, es responsable de promover estilos de vida saludables en la comunidad con el fin de prevenir y controlar factores de riesgo asociados a mayor mortalidad de estos pacientes tales como obesidad, hipertensión arterial y diabetes ${ }^{22-24}$.

El kinesiólogo, además tiene un rol en el ámbito de la gestión, tanto de la información como de la utilización del recurso humano para dar respuesta a la necesidad asistencial ${ }^{25}$. La visión organizacional ha sido uno de los mayores desafíos sanitarios que hemos debido enfrentar como gremio, dejando en evidencia competencias que cada vez son más reconocidas, no solo por todo el personal sanitario, sino también por la sociedad en general.

Por último, la investigación en kinesiología es un área incipiente de gran crecimiento en los últimos años, y que ha motivado la formación continua de muchos profesionales que buscan ejercer un rol científico asociado a su área de desarrollo disciplinar. El surgimiento de esta nueva enfermedad, COVID-19, constituye una oportunidad única para mostrar y aplicar las competencias en investigación científica, debiendo existir una mirada interdisciplinaria que logre dar respuesta a estos nuevos y más complejos escenarios sani$\operatorname{tarios}^{26}$.

La Figura 1 resume los principales roles e intervenciones kinesiológicas en las diferentes fases clínicas de la enfermedad.

\section{Desafíos y proyecciones}

La evolución y término de la pandemia es incierto. Se espera que el desarrollo de una vacuna pueda controlar la propagación de la infección en los próximos años. Sin embargo, la transformación de SARS-CoV-2 en una cepa endémica durante los próximos años o la presencia de nuevos brotes de contagio, denominada "segunda ola de COVID-19", en diferentes países asociado al escaso desarrollo de inmunidad reportado en pacientes recuperados, son factores que demuestran la complejidad de la situación actual y proyectan un número importante de pacientes con COVID-19 durante los próximos años ${ }^{27}$. En este contexto, los desafíos que enfrenta el kinesiólogo son múltiples.

Es necesario el desarrollo e implementación de programas de evaluación e intervención de pacientes con COVID-19 de manera transversal durante todo el curso clínico de la enfermedad, desde las etapas más agudas a las etapas posteriores al alta hospitalaria incluyendo programas que contemplen métodos de evaluación específicos en conjunto con intervenciones individualizadas y protocolizadas ${ }^{26}$.

Por otro lado, se requiere la implementación y gestión de programas de rehabilitación presenciales y a distancia (tele-rehabilitación) coordinados desde los distintos niveles de atención en salud y que den cobertura a las necesidades de nuestra población con el objetivo de contribuir a la reintegración familiar, social y laboral; reducir la morbi-mortalidad, mejorar la calidad de vida y reducir los costos asociados a la atención de salud $^{25}$. 


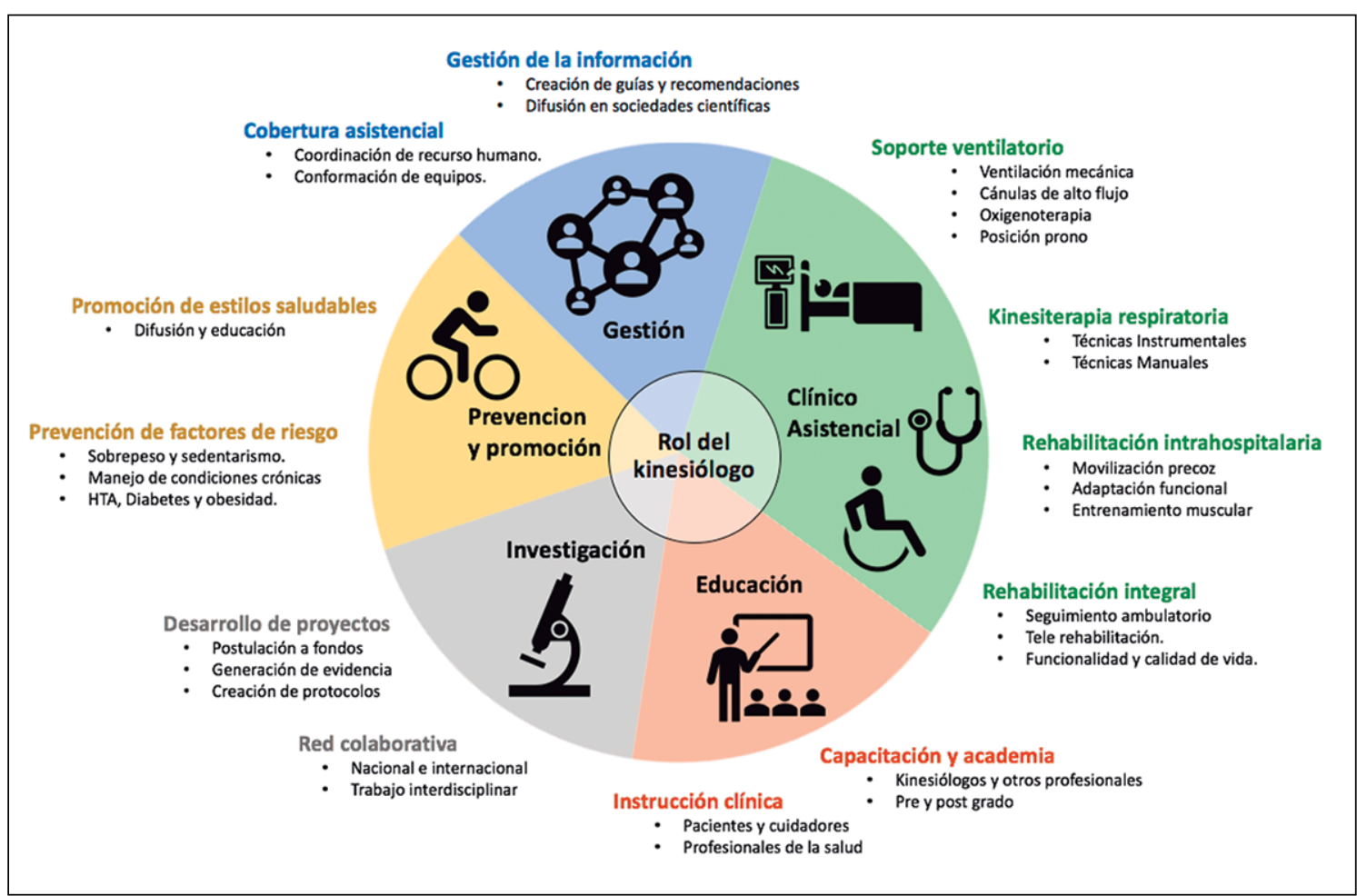

Figura 1. Roles e intervenciones kinesiológicas principales en COVID-19 (HTA: hipertensión arterial).

En educación, resulta esencial crear o potenciar programas existentes de capacitación a los distintos profesionales de salud que profundicen en los conocimientos teóricos y prácticos. Así mismo, el kinesiólogo debe participar activamente en la creación y difusión de guías clínicas, a fin de favorecer el manejo óptimo de estos pacientes. De igual importancia, es razonable considerar una reformulación en los programas de formación de pregrado que incluya diversos aspectos relacionados a la situación sanitaria actual.

Finalmente, resulta trascendental generar un grupo de investigación colaborativo e interdisciplinario con enfoque clínico y traslacional que permita generar redes nacionales e internacionales que promuevan continua y activamente el estudio y desarrollo científico en pacientes que cursen o se hayan recuperado de COVID-19.

\section{Conclusión}

El kinesiólogo posee un rol importante dentro del equipo interdisciplinario de salud y es protagonista en todo el curso clínico de la enfermedad por COVID-19, no solo desde el punto de vista clínico asistencial, sino que además desde su participación en educación, prevención, promoción de estilos saludables, gestión e investigación científica. Es responsabilidad del kinesiólogo apreciar esta oportunidad para aunar esfuerzos y asumir parte de los desafíos actuales, potenciar su rol disciplinar, fortalecer su participación en el equipo de salud y aportar con estrategias eficientes para dar respuesta a las necesidades de nuestra población, hoy mucho más vulnerable debido a la pandemia de COVID-19.

\section{Agradecimientos}

Yorschua Jalil y Roque Basoalto agradecen el aporte parcial entregado por la Agencia $\mathrm{Na}-$ cional de Investigación y Desarrollo/Doctorado Nacional/2019-Folio 21191025 y 2020-Folio 21201751 respectivamente.

\section{Bibliografía}

1.- JOHNS HOPKINS UNIVERSITY. Coronavirus Resource Center. [Internet] 2020. Disponible en: https:// coronavirus.jhu.edu/map.html. 
2.- MINISTERIO DE SALUD DE CHILE. Reporte diario 26 de Julio de 2020. [Internet] 2020. Available from: https://cdn.digital.gob.cl/public_files/Campañas/ Corona-Virus/Reportes/26.07.2020_Reporte_Covid19. pdf

3.- WU Z, MCGOOGAN JM. Characteristics of and Important Lessons From the Coronavirus Disease 2019 (COVID-19) Outbreak in China. JAMA [Internet]. 2020 Apr 7; 323 (13): 1239.Disponible en: https://jamanetwork.com/journals/jama/fullarticle/2762130

4.- GUAN W, NI Z, HU Y, LIANG W, OU C, HE J, et al. Clinical characteristics of coronavirus disease 2019 in China. N Engl J Med. 2020; 382 (18): 1708-20.

5.- VANHOREBEEK I, LATRONICO N, VAN DEN BERGHE G. ICU-acquired weakness. Intensive Care Med. [Internet] 2020; 46 (4): 637-53.Disponible en: https://doi.org/10.1007/s00134-020-05944-4

6.- CURLEY GF, LAFFEY JG, ZHANG H, SLUTSKY AS. Biotrauma and Ventilator-Induced Lung Injury: Clinical Implications. Chest [Internet] 2016; 150 (5): 1109-17.Disponible en: http://dx.doi.org/10.1016/j. chest.2016.07.019

7.- HERRIDGE MS, TANSEY CM, MATTÉ A, TOMLINSON G, DIAZ-GRANADOS N, COOPER A, et al. Functional Disability 5 Years after Acute Respiratory Distress Syndrome. N Engl J Med. [Internet] 2011 Apr 7; 364 (14): 1293-304.Disponible en: http://www.nejm. org/doi/abs/10.1056/NEJMoa1011802

8.- THILLE AW, BOISSIER F, MULLER M, LEVRAT A, BOURDIN G, ROSSELLI S, et al. Role of ICUacquired weakness on extubation outcome among patients at high risk of reintubation. Crit Care. 2020; 24 (1): 1-9.

9.- KRESS JP, HALL JB. ICU-acquired weakness and recovery from critical illness. N Engl J Med. 2014; 370 (17): 1626-35.

10.- CARFÌ A, BERNABEI R, LANDI F. Persistent Symptoms in Patients After Acute COVID-19. JAMA [Internet] 2020; 369: 1-2.Disponible en: https://jamanetwork. com/journals/jama/fullarticle/2768351

11.- MO X, JIAN W, SU Z, CHEN M, PENG H, PENG P, et al. Abnormal pulmonary function in COVID-19 patients at time of hospital discharge. Eur Respir J. 2020; 55 (6): $2-5$.

12.- HERRIDGE MS, MOSS M, HOUGH CL, HOPKINS RO, RICE TW, BIENVENU OJ, et al. Recovery and outcomes after the acute respiratory distress syndrome (ARDS) in patients and their family caregivers. Intensive Care Med. 2016; 42 (5): 725-38.

13.- SIMPSON R, ROBINSON L. Rehabilitation after critical illness in people with COVID-19 infection. Am J Phys Med Rehabil. 2020; 99 (6): 470-4.

14.- MURTHY S, GOMERSALL CD, FOWLER RA. Care for Critically Ill Patients with COVID-19. JAMA - J Am Med Assoc. 2020; 323 (15): 1499-500.

15.- FREDES S, TIRIBELLI N, SETTEN M, RODRI-
GUES R, PLOTNIKOW G, BUSICO M, et al. Definición del rol y las competencias del kinesiólogo en la Unidad de Cuidados Intensivos. Rev Argentina Ter Intensiva [Internet] 2018; 35 (4): 1-10. Disponible en: http://revista.sati.org.ar/index.php/MI/article/view/592

16.- SONG WS, MULLON J, REGAN NA, ROTH BJ. Instruction of hospitalized patients by respiratory therapists on metered-dose inhaler use leads to decrease in patient errors. Respir Care. 2005; 50 (8): 1040-5.

17.- ANDREWS J, SATHE NA, KRISHNASWAMI S, MELISSA L. Nonpharmacologic airway clearance techniques in hospitalized patients: A systematic review. Respir Care. 2013; 58 (12): 2160-86.

18.- VOLPE MS, GUIMARÃES FS, MORAIS CC. Airway Clearance Techniques for Mechanically Ventilated Patients: Insights for Optimization. Respir Care. 2020; 65 (8): 1174-88.

19.- FALVEY JR, KRAFFT C, KORNETTI D. The Essential Role of Home- and Community-Based Physical Therapists During the COVID-19 Pandemic. Phys Ther. 2020; 100 (7): 1058-61.

20.- FELTEN-BARENTSZ KM, VAN OORSOUW R, KLOOSTER E, KOENDERS N, DRIEHUIS F, HULZEBOS EHJ, et al. Recommendations for Hospital-Based Physical Therapists Managing Patients With COVID-19. Phys Ther [Internet] 2020; 100 (9): 1444-57. Disponible en: https://academic.oup.com/ptj/ article/100/9/1444/5859492

21.- LEW HL, LEW HL, OH-PARK M, OH-PARK M, CIFU DX, CIFU DX, et al. The War on COVID-19 Pandemic: Role of Rehabilitation Professionals and Hospitals. Am J Phys Med Rehabil. 2020; 99 (7): 5712.

22.- GUPTA S, HAYEK SS, WANG W, CHAN L, MATHEWS KS, MELAMED ML, et al. Factors Associated With Death in Critically Ill Patients With Coronavirus Disease 2019 in the US. JAMA Intern Med. [Internet] 2020; 2115: 1-11.Disponible en: http:// www.ncbi.nlm.nih.gov/pubmed/32667668

23.- RICHARDSON S, HIRSCH JS, NARASIMHAN M, CRAWFORD JM, MCGINN T, DAVIDSON $\mathrm{KW}$, et al. Presenting Characteristics, Comorbidities, and Outcomes among 5700 Patients Hospitalized with COVID-19 in the New York City Area. JAMA - J Am Med Assoc. 2020; 323 (20): 2052-9.

24.- DEAN E, JONES A, YU HP, GOSSELINK R, SKINNER M. Translating COVID-19 Evidence to Maximize Physical Therapists' Impact and Public Health Response. Phys Ther [Internet]. 2020; 100 (9): 1458-64.Disponible en: https://academic.oup.com/ptj/ article/100/9/1458/5862054

25.- MIDDLETON A, SIMPSON KN, BETTGER JP, BOWDEN MG. COVID-19 Pandemic and Beyond: Considerations and Costs of Telehealth Exercise Programs for Older Adults With Functional Impairments Living at Home-Lessons Learned From a Pilot Case 
Study. Phys Ther. [Internet] 2020; 100 (8): 1278-88. Disponible en: https://academic.oup.com/ptj/article/100/8/1278/5828923

26.- KEENEY T. Physical Therapy in the COVID-19 Pandemic: Forging a Paradigm Shift for Rehabilitation in
Acute Care. Phys Ther. 2020; 100 (8): 1265-7.

27.- THE LANCET. COVID-19: the worst may be yet to come. Lancet (London, England) [Internet] 2020; 396 (10244): 71. Disponible en: http://dx.doi.org/10.1016/ S0140-6736(20)31517-8.

Correspondencia a:

Klgo. Patricio García V.

Departamento Ciencias de la Salud.

Pontificia Universidad Católica de Chile

Vicuña Mackenna 4860, Santiago, Chile.

Email: pgarcs@uc.cl 